\title{
Existence result for a Kirchhoff elliptic system with variable parameters and additive right-hand side via sub- and supersolution method
}

\author{
Mohamed Haiour', Salah Boulaaras ${ }^{2,3 *}$, Youcef Bouizem ${ }^{4}$ and Rafik Guefaifia ${ }^{5}$
}

On the occasion of the 80th birthday of the second author's mother, Mrs. Fatma Bint Al-Tayeb Zeghdoud

"Correspondence:

s.boularas@qu.edu.sa;

saleh_boulaares@yahoo.fr

${ }^{2}$ Department of Mathematics,

College of Sciences and Arts,

Qassim University, Al-Rass, Kingdom of Saudi Arabia

${ }^{3}$ Laboratory of Fundamental and Applied Mathematics of Oran (LMFAO), University of Oran 1, Ahmed Benbella, Algeria

Full list of author information is available at the end of the article

\begin{abstract}
The paper deals with the study of the existence result for a Kirchhoff elliptic system with additive right-hand side and variable parameters by using the sub-/supersolution method. Our study is a natural extension result of our previous one in (Boulaaras and Guefaifia in Math. Methods Appl. Sci. 41:5203-5210, 2018), where we discussed only the simple case when the parameters are constant.
\end{abstract}

MSC: 35J60; 35B30; 35B40

Keywords: Kirchhoff elliptic systems; Existence; Positive solutions; Sub-/supersolution; Multiple parameters

\section{Introduction}

Consider the following system:

$$
\begin{cases}-A\left(\int_{\Omega}|\nabla u|^{2} d x\right) \Delta u=\alpha(x) f(v)+\beta(x) g(u) & \text { in } \Omega, \\ -B\left(\int_{\Omega}|\nabla v|^{2} d x\right) \Delta v=\gamma(x) h(u)+\eta(x) l(v) & \text { in } \Omega, \\ u=v=0 & \text { on } \partial \Omega,\end{cases}
$$

where $\Omega \subset \mathbb{R}^{N}(N \geq 3)$ is a bounded smooth domain with $C^{2}$ boundary $\partial \Omega$, and $A, B$ : $\mathbb{R}^{+} \rightarrow \mathbb{R}^{+}$are continuous functions with further conditions to be given later, $\alpha, \beta, \gamma, \eta \in$ $C(\bar{\Omega})$.

This nonlocal problem originates from the stationary version of Kirchhoff's work [16] in 1883 , namely

$$
\rho \frac{\partial^{2} u}{\partial t^{2}}-\left(\frac{P_{0}}{h}+\frac{E}{2 L} \int_{0}^{L}\left|\frac{\partial u}{\partial x}\right|^{2} d x\right) \frac{\partial^{2} u}{\partial x^{2}}=0
$$

(c) The Author(s) 2020. This article is licensed under a Creative Commons Attribution 4.0 International License, which permits use, sharing, adaptation, distribution and reproduction in any medium or format, as long as you give appropriate credit to the original author(s) and the source, provide a link to the Creative Commons licence, and indicate if changes were made. The images or other third party material in this article are included in the article's Creative Commons licence, unless indicated otherwise in a credit line to the material. If material is not included in the article's Creative Commons licence and your intended use is not permitted by statutory regulation or exceeds the permitted use, you will need to obtain permission directly from the copyright holder. To view a copy of this licence, visit http://creativecommons.org/licenses/by/4.0/. 
where Kirchhoff extended the classical d'Alembert's wave equation by considering the effect of the changes in the length of the string during vibrations. The parameters in (1.2) have the following meanings: $L$ is the length of the string, $h$ is the area of the cross-section, $E$ is the Young modulus of the material, $\rho$ is the mass density, and $P_{0}$ is the initial tension.

Recently, Kirchhoff elliptic equations have been heavily studied, we refer to $[1-7,9,11-$ 15, 17-20].

In [2], Alves and Correa proved the validity of sub-/supersolution method for problems of Kirchhoff class involving a single equation and a boundary condition

$$
\begin{cases}-M\left(\|u\|^{2}\right) \Delta u=f(x, u) & \text { in } \Omega, \\ u=0 & \text { on } \partial \Omega\end{cases}
$$

with $f \in C(\bar{\Omega} \times \mathbb{R})$.

By using a comparison principle that requires $M$ to be nonnegative and nonincreasing in $[0,+\infty)$, with $H(t):=M\left(t^{2}\right) t$ increasing and $H(\mathbb{R})=\mathbb{R}$, they managed to prove the existence of positive solutions assuming $f$ was increasing in $u$ for each $x \in \Omega$ fixed.

For systems involving similar equations, this result cannot be used directly, i.e., the existence of a subsolution and a supersolution does not guarantee the existence of the solution. Therefore, a further construction is needed. In [8], we studied the system

$$
\begin{cases}-A\left(\int_{\Omega}|\nabla u|^{2} d x\right) \Delta u=\lambda_{1} f(v)+\mu_{1} g(u) & \text { in } \Omega, \\ -B\left(\int_{\Omega}|\nabla v|^{2} d x\right) \Delta v=\lambda_{2} h(u)+\mu_{2}(x) l(v) & \text { in } \Omega, \\ u=v=0 & \text { on } \partial \Omega .\end{cases}
$$

Using a weak positive supersolution as the first term of a constructed iterative sequence $\left(u_{n}, v_{n}\right)$ in $H_{0}^{1}(\Omega) \times H_{0}^{1}(\Omega)$, and a comparison principle introduced in [2], the authors established the convergence of this sequence to a positive weak solution of the considered problem.

To complement our above work in [8], where we discussed only the simple case when the parameters are constant, in this paper we prove an existence result for problem (1.1) by considering the complicated case when the parameters $\alpha, \beta, \gamma$, and $\eta$ on the righthand side are variable. We also give a better subsolution providing easier computations compared with the earlier work in [8].

\section{Existence result}

Definition 1 A pair $(u, v) \in\left(H_{0}^{1}(\Omega) \times H_{0}^{1}(\Omega)\right)$ is called a weak solution of (1.1) if it satisfies

$$
\begin{aligned}
& A\left(\int_{\Omega}|\nabla u|^{2} d x\right) \int_{\Omega} \nabla u \nabla \phi d x=\int_{\Omega} \alpha(x) f(v) \phi d x+\int_{\Omega} \beta(x) g(u) \phi d x \text { in } \Omega, \\
& B\left(\int_{\Omega}|\nabla v|^{2} d x\right) \int_{\Omega} \nabla v \nabla \psi d x=\int_{\Omega} \gamma(x) h(u) \psi d x+\int_{\Omega} \eta(x) l(v) \psi d x \text { in } \Omega
\end{aligned}
$$

for all $(\phi, \psi) \in\left(H_{0}^{1}(\Omega) \times H_{0}^{1}(\Omega)\right)$.

Definition 2 Let $(\underline{u}, \underline{v}),(\bar{u}, \bar{v})$ be pairs of nonnegative functions in $\left(H_{0}^{1}(\Omega) \times H_{0}^{1}(\Omega)\right)$. They are called a positive weak subsolution and a positive weak supersolution, respectively, of 
(1.1) if they satisfy the following:

$$
\begin{aligned}
& A\left(\int_{\Omega}|\nabla \underline{u}|^{2} d x\right) \int_{\Omega} \nabla \underline{u} \nabla \phi d x \leq \int_{\Omega} \alpha(x) f(\underline{v}) \phi d x+\int_{\Omega} \beta(x) g(\underline{u}) \phi d x, \\
& B\left(\int_{\Omega}|\nabla \underline{v}|^{2} d x\right) \int_{\Omega} \nabla \underline{v} \nabla \psi d x \leq \int_{\Omega} \gamma(x) h(\underline{u}) \psi d x+\int_{\Omega} \eta(x) l(\underline{v}) \psi d x,
\end{aligned}
$$

and

$$
\begin{aligned}
& A\left(\int_{\Omega}|\nabla \bar{u}|^{2} d x\right) \int_{\Omega} \nabla \bar{u} \nabla \phi d x \geq \int_{\Omega} \alpha(x) f(\bar{v}) \phi d x+\int_{\Omega} \beta(x) g(\bar{u}) \phi d x, \\
& B\left(\int_{\Omega}|\nabla \bar{v}|^{2} d x\right) \int_{\Omega} \nabla \bar{v} \nabla \psi d x \geq \int_{\Omega} \gamma(x) h(\bar{u}) \psi d x+\int_{\Omega} \eta(x) l(\bar{v}) \psi d x
\end{aligned}
$$

for all $(\phi, \psi) \in\left(H_{0}^{1}(\Omega) \times H_{0}^{1}(\Omega)\right)$, with $\phi \geq 0$ and $\psi \geq 0$, and $(\underline{u}, \underline{v}),(\bar{u}, \bar{v})=(0,0)$ on $\partial \Omega$.

Lemma 1 (Comparison principle [2]) Let $M: \mathbb{R}^{+} \rightarrow \mathbb{R}^{+}$be a continuous nonincreasing function such that

$$
M(s)>m_{0}>0, \text { for all } s \geq s_{0},
$$

and $H(t)=t M\left(t^{2}\right)$ increasing on $\mathbb{R}^{+}$.

If $u_{1}, u_{2}$ are two nonnegative functions verifying

$$
\begin{cases}-M\left(\int_{\Omega}\left|\nabla u_{1}\right|^{2} d x\right) \Delta u_{1} \geq-M\left(\int_{\Omega}\left|\nabla u_{2}\right|^{2} d x\right) \Delta u_{2} & \text { in } \Omega, \\ u=v=0 & \text { on } \partial \Omega,\end{cases}
$$

then $u_{1} \geq u_{2}$ a.e. in $\Omega$.

Before stating and proving our main result, here are the conditions we need:

(H1) $A, B: \mathbb{R}^{+} \rightarrow \mathbb{R}^{+}$are two continuous and increasing functions that satisfy the monotonicity conditions of Lemma 1 so that we can use the comparison principle, and assume further that there exist $a_{i}, b_{i}>0, i=1,2$,

$$
a_{1} \leq A(t) \leq a_{2}, \quad b_{1} \leq B(t) \leq b_{2} \quad \text { for all } t \in \mathbb{R}^{+}
$$

(H2) $\alpha, \beta, \gamma, \eta \in C(\bar{\Omega})$ and

$$
\alpha(x) \geq \alpha_{0}>0, \quad \beta(x) \geq \beta_{0}>0, \quad \gamma(x) \geq \gamma_{0}>0, \quad \eta(x) \geq \eta_{0}>0
$$

for all $x \in \Omega$.

(H3) $f, g, h$, and $l$ are continuous on $\left[0,+\infty\left[, C^{1}\right.\right.$ on $(0,+\infty)$, and increasing functions of infinite growth

$$
\lim _{t \rightarrow+\infty} f(t)=+\infty, \quad \lim _{t \rightarrow+\infty} l(t)=+\infty, \quad \lim _{t \rightarrow+\infty} g(t)=+\infty, \quad \lim _{t \rightarrow+\infty} h(t)=+\infty
$$


(H4) For all $K>0$,

$$
\lim _{t \rightarrow+\infty} \frac{f(K(h(t)))}{t}=0
$$

(H5)

$$
\lim _{t \rightarrow+\infty} \frac{g(t)}{t}=\lim _{t \rightarrow+\infty} \frac{l(t)}{t}=0
$$

Theorem 1 For large values of $\alpha_{0}+\beta_{0}$ and $\gamma_{0}+\eta_{0}$, system (1.1) admits a large positive weak solution if conditions (H1)-(H5) are satisfied.

Proof of Theorem 1 Consider $\sigma$ to be the first eigenvalue of $-\Delta$ with Dirichlet boundary conditions and $\phi_{1}$ the corresponding positive eigenfunction with $\left\|\phi_{1}\right\|=1$ and $\phi_{1} \in$ $C^{\infty}(\bar{\Omega})$ (see $\left.[10]\right)$.

Let $S=\sup _{x \in \Omega}\left\{\sigma \phi_{1}^{2}-\left|\nabla \phi_{1}\right|^{2}\right\}$, then from growth condition (H3)

$$
f(t) \geq S, \quad g(t) \geq S, \quad h(t) \geq S, \quad l(t) \geq S, \quad \text { for } t \text { large enough. }
$$

For each $\alpha_{0}+\beta_{0}$ and $\gamma_{0}+\eta_{0}$ large, let us define

$$
\underline{u}=\frac{\alpha_{0}+\beta_{0}}{2 a_{2}} \phi_{1}^{2}
$$

and

$$
\underline{v}=\frac{\gamma_{0}+\eta_{0}}{2 b_{2}} \phi_{1}^{2}
$$

where $a_{2}, b_{2}$ are given by condition (H1). Let us show that $(\underline{u}, \underline{v})$ is a subsolution of problem (1.1) for $\alpha_{0}+\beta_{0}$ and $\gamma_{0}+\eta_{0}$ large enough. Indeed, let $\phi \in H_{0}^{1}(\Omega)$ with $\phi \geq 0$ in $\Omega$. By (H1)(H3), we get

$$
\begin{aligned}
A\left(\int_{\Omega}|\nabla \underline{u}|^{2} d x\right) \int_{\Omega} \nabla \underline{u} \nabla \phi d x= & A\left(\int_{\Omega}|\nabla \underline{u}|^{2} d x\right) \frac{\alpha_{0}+\beta_{0}}{a_{2}} \int_{\Omega} \phi_{1} \nabla \phi_{1} \nabla \phi d x \\
= & \frac{\alpha_{0}+\beta_{0}}{a_{2}} A\left(\int_{\Omega}|\nabla \underline{u}|^{2} d x\right) \\
& \times\left\{\int_{\Omega} \nabla \phi_{1} \nabla\left(\phi_{1} \phi\right) d x-\int_{\Omega}\left|\nabla \phi_{1}\right|^{2} \phi d x\right\} \\
= & \frac{\alpha_{0}+\beta_{0}}{a_{2}} A\left(\int_{\Omega}|\nabla \underline{u}|^{2} d x\right) \int_{\Omega}\left(\sigma \phi_{1}^{2}-\left|\nabla \phi_{1}\right|^{2}\right) \phi d x \\
\leq & \left(\alpha_{0}+\beta_{0}\right) \int_{\Omega} S \phi d x \\
\leq & \int_{\Omega} \alpha(x) f(\underline{v}) \phi d x+\int_{\Omega} \beta(x) g(\underline{u}) \phi d x
\end{aligned}
$$

for $\alpha_{0}+\beta_{0}>0$ large enough, and all $\phi \in H_{0}^{1}(\Omega)$ with $\phi \geq 0$ in $\Omega$. 
Similarly,

$$
B\left(\int_{\Omega}|\nabla \underline{v}|^{2} d x\right) \int_{\Omega} \nabla \underline{v} \nabla \psi d x \leq \int_{\Omega} \gamma(x) h(\underline{u}) \psi d x+\int_{\Omega} \eta(x) \mathrm{t}(\underline{v}) \psi d x \quad \text { in } \Omega
$$

for $\gamma_{0}+\eta_{0}>0$ large enough and all $\psi \in H_{0}^{1}(\Omega)$ with $\psi \geq 0$ in $\Omega$.

Also notice that $\underline{u}>0$ and $\underline{v}>0$ in $\Omega, \underline{u} \rightarrow+\infty$ and $\underline{v} \rightarrow+\infty$ as $\alpha_{0}+\beta_{0} \rightarrow+\infty$ and $\gamma_{0}+\eta_{0} \rightarrow+\infty$.

For the supersolution part, consider $e$ the solution of the following problem:

$$
\begin{cases}-\triangle e=1 & \text { in } \Omega, \\ e=0 & \text { on } \partial \Omega .\end{cases}
$$

We give the supersolution of problem (1.1) by

$$
\bar{u}=C e, \quad \bar{v}=\left(\|\gamma\|_{\infty}+\|\eta\|_{\infty}\right) h\left(C\|e\|_{\infty}\right) e,
$$

where $C>0$ is a large positive real number to be given later.

Indeed, for all $\phi \in H_{0}^{1}(\Omega)$ with $\phi \geq 0$ in $\Omega$, we get from (2.3) and the condition (H1)

$$
\begin{aligned}
A\left(\int_{\Omega}|\nabla \bar{u}|^{2} d x\right) \int_{\Omega} \nabla \bar{u} \nabla \phi d x & =A\left(\int_{\Omega}|\nabla \bar{u}|^{2} d x\right) C \int_{\Omega} \nabla e \nabla \phi d x \\
& =A\left(\int_{\Omega}|\nabla \bar{u}|^{2} d x\right) C \int_{\Omega} \phi d x \\
& \geq a_{1} C \int_{\Omega} \phi d x .
\end{aligned}
$$

By (H4) and (H5), we can choose $C$ large enough so that

$$
a_{1} C \geq\|\alpha\|_{\infty} f\left[\left(\|\gamma\|_{\infty}+\|\eta\|_{\infty}\right) h\left(C\|e\|_{\infty}\right)\|e\|_{\infty}\right]+\|\beta\|_{\infty} g\left(C\|e\|_{\infty}\right) .
$$

Therefore,

$$
\begin{aligned}
& A\left(\int_{\Omega}|\nabla \bar{u}|^{2} d x\right) \int_{\Omega} \nabla \bar{u} . \nabla \phi d x \\
& \quad \geq\left[\|\alpha\|_{\infty} f\left[\left(\|\gamma\|_{\infty}+\|\eta\|_{\infty}\right) h\left(C\|e\|_{\infty}\right)\|e\|_{\infty}\right]+\|\beta\|_{\infty} g\left(C\|e\|_{\infty}\right)\right] \int_{\Omega} \phi d x \\
& \quad \geq\|\alpha\|_{\infty} \int_{\Omega} f\left[\left(\|\gamma\|_{\infty}+\|\eta\|_{\infty}\right) h\left(C\|e\|_{\infty}\right)\|e\|_{\infty}\right] \phi d x+\|\beta\|_{\infty} \int_{\Omega} g\left(C\|e\|_{\infty}\right) \phi d x \\
& \quad \geq \int_{\Omega} \alpha(x) f(\bar{v}) \phi d x+\int_{\Omega} \beta(x) g(\bar{u}) \phi d x .
\end{aligned}
$$

Also,

$$
\begin{aligned}
B\left(\int_{\Omega}|\nabla \bar{\nu}|^{2} d x\right) \int_{\Omega} \nabla \bar{\nu} \nabla \psi d x & =\left(\|\gamma\|_{\infty}+\|\eta\|_{\infty}\right) \int_{\Omega} h\left(C\|e\|_{\infty}\right) \psi d x \\
& \geq \int_{\Omega} \gamma(x) h(\bar{u}) \psi d x+\int_{\Omega} \eta(x) h\left(C\|e\|_{\infty}\right) \psi d x
\end{aligned}
$$


Using (H4) and (H5) again for $C$ large enough, we get

$$
h\left(C\|e\|_{\infty}\right) \geq l\left[\left(\|\gamma\|_{\infty}+\|\eta\|_{\infty}\right) h\left(C\|e\|_{\infty}\right)\|e\|_{\infty}\right] \geq l(\bar{v}) .
$$

Combining (2.5) and (2.6), we obtain

$$
B\left(\int_{\Omega}|\nabla \bar{v}|^{2} d x\right) \int_{\Omega} \nabla \bar{v} \nabla \psi d x \geq \int_{\Omega} \gamma(x) h(\bar{u}) \psi d x+\int_{\Omega} \eta(x) l(\bar{v}) \psi d x .
$$

By (2.4) and (2.7), we conclude that $(\bar{u}, \bar{v})$ is a supersolution of problem (1.1).

Furthermore, $\underline{u} \leq \bar{u}$ and $\underline{v} \leq \bar{v}$ for $C$ chosen large enough.

Now, we use a similar argument to that in [8] in order to obtain a weak solution of our problem. Consider the following sequence $\left\{\left(u_{n}, v_{n}\right)\right\} \subset\left(H_{0}^{1}(\Omega) \times H_{0}^{1}(\Omega)\right)$ where $u_{0}:=\bar{u}$, $v_{0}=\bar{v}$, and $\left(u_{n}, v_{n}\right)$ is the unique solution of

$$
\begin{cases}-A\left(\int_{\Omega}\left|\nabla u_{n}\right|^{2} d x\right) \Delta u_{n}=\alpha(x) f\left(v_{n-1}\right)+\beta(x) g\left(u_{n-1}\right) & \text { in } \Omega, \\ -B\left(\int_{\Omega}\left|\nabla v_{n}\right|^{2} d x\right) \Delta v_{n}=\gamma(x) h\left(u_{n-1}\right)+\eta(x) l\left(v_{n-1}\right) & \text { in } \Omega, \\ u_{n}=v_{n}=0 & \text { on } \partial \Omega .\end{cases}
$$

Since $A$ and $B$ satisfy (H1) and $\alpha(x) f\left(v_{n-1}\right), \beta(x) g\left(u_{n-1}\right), \gamma(x) h\left(u_{n-1}\right)$, and $\eta(x) l\left(v_{n-1}\right) \in$ $L^{2}(\Omega)$ (in $\left.x\right)$, we deduce from a result in [2] that system (2.8) has a unique solution $\left(u_{n}, v_{n}\right) \in$ $\left(H_{0}^{1}(\Omega) \times H_{0}^{1}(\Omega)\right)$.

Using (2.8) and the fact that $\left(u_{0}, v_{0}\right)$ is a supersolution of $(1.1)$, we get

$$
\left\{\begin{array}{l}
-A\left(\int_{\Omega}\left|\nabla u_{0}\right|^{2} d x\right) \Delta u_{0} \geq \alpha(x) f\left(v_{0}\right)+\beta(x) g\left(u_{0}\right)=-A\left(\int_{\Omega}\left|\nabla u_{1}\right|^{2} d x\right) \Delta u_{1}, \\
-B\left(\int_{\Omega}\left|\nabla v_{0}\right|^{2} d x\right) \triangle v_{0} \geq \gamma(x) h\left(u_{0}\right)+\eta(x) l\left(v_{0}\right)=-B\left(\int_{\Omega}\left|\nabla v_{1}\right| d x\right) \triangle v_{1} .
\end{array}\right.
$$

Then by Lemma $1, u_{0} \geq u_{1}$ and $v_{0} \geq v_{1}$. Also, since $u_{0} \geq \underline{u}, v_{0} \geq \underline{v}$ and due to the monotonicity of $f, g, h$, and $l$, one has

$$
\begin{aligned}
-A\left(\int_{\Omega}\left|\nabla u_{1}\right|^{2} d x\right) \triangle u_{1} & =\alpha(x) f\left(v_{0}\right)+\beta(x) g\left(u_{0}\right) \\
& \geq \alpha(x) f(\underline{v})+\beta(x) g(\underline{u}) \geq-A\left(\int_{\Omega}|\nabla \underline{u}|^{2} d x\right) \triangle \underline{u}, \\
-B\left(\int_{\Omega}\left|\nabla v_{1}\right|^{2} d x\right) \triangle v_{1} & =\gamma(x) h\left(u_{0}\right)+\eta(x) l\left(v_{0}\right) \\
& \geq \gamma(x) h(\underline{u})+\eta(x) l(\underline{v}) \geq-B\left(\int_{\Omega}|\nabla \underline{v}|^{2} d x\right) \triangle \underline{v} .
\end{aligned}
$$

According to Lemma 1 again, we obtain $u_{1} \geq \underline{u}, v_{1} \geq \underline{v}$.

Repeating the same argument for $u_{2}, v_{2}$, observe that

$$
\begin{aligned}
-A\left(\int_{\Omega}\left|\nabla u_{1}\right|^{2} d x\right) \triangle u_{1} & =\alpha(x) f\left(v_{0}\right)+\beta(x) g\left(u_{0}\right) \\
& \geq \alpha(x) f\left(v_{1}\right)+\beta(x) g\left(u_{1}\right)=-A\left(\int_{\Omega}\left|\nabla u_{2}\right|^{2} d x\right) \triangle u_{2},
\end{aligned}
$$




$$
\begin{aligned}
-B\left(\int_{\Omega}\left|\nabla v_{1}\right| d x\right) \Delta v_{1} & =\gamma(x) h\left(u_{0}\right)+\eta(x) l\left(v_{0}\right) \\
& \geq \gamma(x) h\left(u_{1}\right)+\eta(x) l\left(v_{1}\right)=-B\left(\int_{\Omega}\left|\nabla v_{2}\right|^{2} d x\right) \triangle v_{2},
\end{aligned}
$$

and then $u_{1} \geq u_{2}, v_{1} \geq v_{2}$. Similarly, we get $u_{2} \geq \underline{u}$ and $v_{2} \geq \underline{v}$ from

$$
\begin{aligned}
-A\left(\int_{\Omega}\left|\nabla u_{2}\right|^{2} d x\right) \triangle u_{2} & =\alpha(x) f\left(v_{1}\right)+\beta(x) g\left(u_{1}\right) \\
& \geq \alpha(x) f(\underline{v})+\beta(x) g(\underline{u}) \geq-A\left(\int_{\Omega}|\nabla \underline{u}|^{2} d x\right) \triangle \underline{u}, \\
-B\left(\int_{\Omega}\left|\nabla v_{2}\right|^{2} d x\right) \triangle v_{2} & =\gamma(x) h\left(u_{1}\right)+\eta(x) l\left(v_{1}\right) \\
& \geq \gamma(x) h(\underline{u})+\eta(x) l(\underline{v}) \geq-B\left(\int_{\Omega}|\nabla \underline{v}|^{2} d x\right) \triangle \underline{v} .
\end{aligned}
$$

By repeating the same arguments, we construct a bounded decreasing sequence $\left\{\left(u_{n}, v_{n}\right)\right\} \subset\left(H_{0}^{1}(\Omega) \times H_{0}^{1}(\Omega)\right)$ verifying

$$
\begin{aligned}
& \bar{u}=u_{0} \geq u_{1} \geq u_{2} \geq \cdots \geq u_{n} \geq \cdots \geq \underline{u}>0, \\
& \bar{v}=v_{0} \geq v_{1} \geq v_{2} \geq \cdots \geq v_{n} \geq \cdots \geq \underline{v}>0 .
\end{aligned}
$$

By continuity of functions $f, g, h$, and $l$ and the definition of the sequences $\left(u_{n}\right)$ and $\left(v_{n}\right)$, there exist positive constants $C_{i}>0, i=1, \ldots, 4$ such that

$$
\left|f\left(v_{n-1}\right)\right| \leq C_{1}, \quad\left|g\left(u_{n-1}\right)\right| \leq C_{2}, \quad\left|h\left(u_{n-1}\right)\right| \leq C_{3}
$$

and

$$
\left|l\left(u_{n-1}\right)\right| \leq C_{4} \text { for all } n
$$

From (2.11), multiplying the first equation of (2.8) by $u_{n}$, integrating, using Hölder inequality and Sobolev embedding, we check that

$$
\begin{aligned}
a_{1} \int_{\Omega}\left|\nabla u_{n}\right|^{2} d x & \leq A\left(\int_{\Omega}\left|\nabla u_{n}\right|^{2} d x\right) \int_{\Omega}\left|\nabla u_{n}\right|^{2} d x \\
& =\int_{\Omega} \alpha(x) f\left(v_{n-1}\right) u_{n} d x+\int_{\Omega} \beta(x) g\left(u_{n-1}\right) u_{n} d x \\
& \leq\|\alpha\|_{\infty} \int_{\Omega}\left|f\left(v_{n-1}\right)\right|\left|u_{n}\right| d x+\|\beta\|_{\infty} \int_{\Omega}\left|g\left(u_{n-1}\right)\right|\left|u_{n}\right| d x \\
& \leq C_{1} \int_{\Omega}\left|u_{n}\right| d x+C_{2} \int_{\Omega}\left|u_{n}\right| d x \\
& \leq C_{5}\left\|u_{n}\right\|_{H_{0}^{1}(\Omega)}
\end{aligned}
$$

or

$$
\left\|u_{n}\right\|_{H_{0}^{1}(\Omega)} \leq C_{5}, \quad \forall n
$$


where $C_{5}>0$ is a constant independent of $n$. Similarly, there exists $C_{6}>0$ independent of $n$ such that

$$
\left\|v_{n}\right\|_{H_{0}^{1}(\Omega)} \leq C_{6}, \quad \forall n
$$

From (2.12) and (2.13), we deduce that $\left\{\left(u_{n}, v_{n}\right)\right\}$ admits a weakly converging subsequence in $H_{0}^{1}\left(\Omega, \mathbb{R}^{2}\right)$ to a limit $(u, v)$ satisfying $u \geq \underline{u}>0$ and $v \geq \underline{v}>0$. Being monotone, by using a standard regularity argument, $\left\{\left(u_{n}, v_{n}\right)\right\}$ converges itself to $(u, v)$. Now, letting $n \rightarrow+\infty$ in (2.8), we conclude that $(u, v)$ is a positive weak solution of system (1.1).

\section{Acknowledgements}

The authors are grateful to the anonymous referees for the careful reading and their important observations/suggestions for the sake of improving this paper. Moreover, the second author would like to thank his Professors/Scientists: Pr. Mohamed Haiour, Pr. Ahmed-Salah Chibi, and Pr. Azzedine Benchettah at Annaba University in Algeria for the important content of Master's and PhD courses in pure and applied mathematics which he received during his studies. Moreover, he thanks them for the additional help they provided him during office hours regarding the few concepts/difficulties he had encountered, and he appreciates their talent and dedication to their postgraduate students currently and previously!

\section{Funding}

Not applicable.

Availability of data and materials

Not applicable.

Ethics approval and consent to participate

Not applicable.

\section{Competing interests}

The authors declare that there is no conflict of interests regarding the publication of this manuscript. The authors declare that they have no competing interests.

\section{Consent for publication}

Not applicable.

\section{Authors' contributions}

The authors contributed equally to this article. They have all read and approved the final manuscript.

\section{Author details}

${ }^{1}$ LANOS Laboratory, Faculty of Sciences, Badji Mokhtar-Annaba University, Annaba 23000, Algeria. ${ }^{2}$ Department of Mathematics, College of Sciences and Arts, Qassim University, Al-Rass, Kingdom of Saudi Arabia. ${ }^{3}$ Laboratory of Fundamental and Applied Mathematics of Oran (LMFAO), University of Oran 1, Ahmed Benbella, Algeria. ${ }^{4}$ Department of Mathematics, Faculty of Mathematics and Informatics, USTOMB, Oran, Algeria. ${ }^{5}$ Department of Mathematics and Computer Science, Larbi Tebessi University, Tebessa, Algeria.

\section{Publisher's Note}

Springer Nature remains neutral with regard to jurisdictional claims in published maps and institutional affiliations.

Received: 1 June 2020 Accepted: 31 July 2020 Published online: 05 August 2020

\section{References}

1. Alizadeh, M., Alimohammady, M.: Regularity and entropy solutions of some elliptic equations. Miskolc Math. Notes $19(2), 715-729(2018)$

2. Alves, C.O., Correa, F.J.S.A.: On existence of solutions for a class of problem involving a nonlinear operator. Commun. Appl. Nonlinear Anal. 8, 43-56 (2001)

3. Azouz, N., Bensedik, A.: Existence result for an elliptic equation of Kirchhoff-type with changing sign data. Funkc Ekvacioj 55, 55-66 (2012)

4. Bouizem, Y., Boulaaras, S., Djebbar, B.: Some existence results for an elliptic equation of Kirchhoff-type with changing sign data and a logarithmic nonlinearity. Math. Methods Appl. Sci. 42(7), 2465-2474 (2019)

5. Bouizem, Y., Boulaaras, S., Djebbar, B.: Existence of positive solutions for a class of Kirchhoff elliptic systems with right hand side defined as a multiplication of two separate functions. Kragujev. J. Math. 45(4), 587-596 (2021)

6. Boulaaras, S.: Existence of positive solutions for a new class of parabolic Kirchhoff systems with right hand side defined as a multiplication of two separate functions. Rocky Mt. J. Math. 50(2), 445-454 (2020)

7. Boulaaras, S.: Existence of positive solutions of nonlocal $\mathrm{p}(\mathrm{x})$-Kirchhoff hyperbolic systems via sub-super solutions concept. J. Intell. Fuzzy Syst. 38(4), 4301-4313 (2020) 
8. Boulaaras, S., Guefaifia, R.: Existence of positive weak solutions for a class of Kirchhoff elliptic systems with multiple parameters. Math. Methods Appl. Sci. 41, 5203-5210 (2018)

9. Correa, F..S.A., Figueiredo, G.M.: On a p-Kirchhoff equation type via Krasnoselkii's genus. Appl. Math. Lett. 22, 819-822 (2009)

10. Evans, L.: Partial Differential Equations. American Mathematical Society, Providence (2010)

11. Fan, X.L., Zhao, D.: On the spaces $L^{p(x)}(\Omega)$ and $W^{m, p(x)}(\Omega)$. J. Math. Anal. Appl. 263, 424-446 (2001)

12. Figueiredo, G.M., Suarez, A.: Some remarks on the comparison principle in Kirchhoff equations. Rev. Mat. Iberoam. 34, 609-620 (2018)

13. Gala, S., Liu, Q., Ragusa, M.A.: A new regularity criterion for the nematic liquid crystal flows. Appl. Anal. 91(9), $1741-1747$ (2012)

14. Gala, S., Ragusa, M.A.: Logarithmically improved regularity criterion for the Boussinesq equations in Besov spaces with negative indices. Appl. Anal. 95(6), 1271-1279 (2016)

15. Garcia-Melian, J., Iturriaga, L.: Some counter examples related to the stationary Kirchhoff equation. Proc. Am. Math. Soc. 144, 3405-3411 (2016)

16. Kirchhoff, G.: Mechanik. Teubner, Leipzig (1883)

17. Ma, T.F:: Remarks on an elliptic equation of Kirchhoff type. Nonlinear Anal. 63, 1967-1977 (2005)

18. Polidoro, S., Ragusa, M.A.: Harnack inequality for hypoelliptic ultraparabolic equations with a singular lower order term. Rev. Mat. Iberoam. 24(3), 1011-1046 (2008)

19. Ricceri, B.: On an elliptic Kirchhoff-type problem depending on two parameters. J. Glob. Optim. 46, 543-549 (2010)

20. Sun, J.J., Tang, C.L.: Existence and multiplicity of solutions for Kirchhoff type equations. Nonlinear Anal. 74, 1212-1222 (2011)

\section{Submit your manuscript to a SpringerOpen ${ }^{\circ}$ journal and benefit from:}

- Convenient online submission

- Rigorous peer review

- Open access: articles freely available online

- High visibility within the field

- Retaining the copyright to your article 